\title{
First-principles study of ternary fcc solution phases from special quasirandom structures
}

\author{
Dongwon Shin, ${ }^{1, *}$ Axel van de Walle, ${ }^{2}$ Yi Wang, ${ }^{1}$ and Zi-Kui Liu ${ }^{1}$ \\ ${ }^{1}$ Department of Materials Science and Engineering, The Pennsylvania State University, University Park, Pennsylvania 16802, USA \\ ${ }^{2}$ Engineering and Applied Science Division, California Institute of Technology, Pasadena, California 91125, USA
}

(Received 1 June 2007; revised manuscript received 17 August 2007; published 16 October 2007)

\begin{abstract}
In the present work, ternary special quasirandom structures (SQSs) for a fcc solid solution phase are generated at different compositions, $x_{A}=x_{B}=x_{C}=\frac{1}{3}$ and $x_{A}=\frac{1}{2}, x_{B}=x_{C}=\frac{1}{4}$, whose correlation functions are satisfactorily close to those of a random fcc solution. The generated SQSs are used to calculate the mixing enthalpy of the fcc phase in the $\mathrm{Ca}-\mathrm{Sr}-\mathrm{Yb}$ system. It is observed that first-principles calculations of all the binary and ternary SQSs in the Ca-Sr-Yb system exhibit very small local relaxation. It is concluded that the fcc ternary SQSs can provide valuable information about the mixing behavior of the fcc ternary solid solution phase. The SQSs presented in this work can be widely used to study the behavior of ternary fcc solid solutions.
\end{abstract}

DOI: 10.1103/PhysRevB.76.144204

PACS number(s): 61.66.Dk, 81.05.Bx

\section{INTRODUCTION}

In calculation of phase diagrams (CALPHAD) modeling, ${ }^{1,2}$ thermodynamic properties of a solution phase in a ternary-or higher-order-system are usually obtained through combining those of its constitutive binary systems and occasionally with ternary interaction parameters. Since the most dominant interatomic reaction in a multicomponent system is that of the binaries, accurate thermodynamic descriptions which are capable of reproducing the characteristics of binary solution phases are prerequisites to a successful multicomponent thermodynamic modeling. In this regard, considerable efforts have been made to develop models for combining thermodynamic descriptions for binary solution phases to be used in higher-order systems. ${ }^{3-8}$

In typical thermodynamic modelings, ternary interaction parameters for solid solution phases are not introduced since their thermochemical data, such as mixing enthalpy, which are needed in the parameter evaluation are very difficult to measure. In fact, obtaining accurate thermochemical data for solid solution phases is challenging even for binaries due to sluggish kinetics at low temperatures, so that it is hard to reach a complete thermodynamic equilibrium. Furthermore, the existence of intermediate phases narrows the experimentally accessible composition range for thermochemical properties of solid solution phases. As the number of elements increases in a multicomponent system, the complexity of acquiring reliable data also increases. Consequently, interaction parameters for the excess Gibbs energy of binary solid solution phases are usually evaluated only from phase equilibrium data and those of ternary are usually omitted due to the lack of data.

Fortunately, the dearth of experimental data for solid solution phases can be overcome by atomic level calculations, such as first-principles calculations. Especially designed supercells, the so-called special quasirandom structures (SQSs) suggested by Zunger et al., ${ }^{9}$ whose lattice sites are occupied by constituent atoms so as to reproduce the average correlation functions of a completely random solution, are one of the representative methods. SQSs can be completely relaxed within density functional theory (DFT) codes to consider the effect of local relaxation and can also be applied to any other system by changing the atoms because they are structural templates. A limitation is that SQSs of a unit cell size that is manageable with present DFT codes can only be obtained at certain compositions, e.g., $x=0.25,0.5$, and 0.75 in the substitutional $A_{1-x} B_{x}$ binary alloys. Nevertheless, first-principles study of SQSs at those three compositions permits accurate predictions of various properties of solid solutions. It has already been successfully applied to calculate thermodynamic properties of binary solid solution phases for fcc, bcc, and hcp phases..$^{10-12}$

Two ternary fcc SQSs in an $A-B-C$ system, which can be applied to four different compositions $\left(x_{A}=x_{B}=x_{C}=\frac{1}{3} ; x_{A}=\frac{1}{2}\right.$, $x_{B}=x_{C}=\frac{1}{4} ; x_{A}=\frac{1}{4}, x_{A}=\frac{1}{2}, x_{C}=\frac{1}{4} ;$ and $\left.x_{A}=x_{B}=\frac{1}{4}, x_{C}=\frac{1}{2}\right)$, are developed to investigate the mixing enthalpies for ternary fcc solid solution phases in the present work. The organization of this paper is as follows. The impact of ternary interaction parameters on a ternary solution phase in the CALPHAD approach is briefly reviewed. Then, the generated ternary fcc SQSs are characterized in terms of their atomic arrangements to reproduce the pair and multisite correlation functions of completely random fcc solid solutions. Finally, the generated SQSs are applied to the Ca-Sr-Yb system, which presumably has fcc solid solution phases throughout the entire composition range in all three binaries and ternary.

\section{TERNARY INTERACTION PARAMETERS}

The Gibbs energy of a ternary solution phase $\phi$ is expressed as $^{6}$

$$
G^{\phi}=\sum_{i=1}^{c} x_{i} G_{i}^{o, \phi}+R T \sum_{i=1}^{c} x_{i} \ln x_{i}+{ }^{x s} G^{\mathrm{bin}, \phi}+{ }^{x s} G^{\mathrm{tern}, \phi},
$$

where $x_{i}$ is the mole fraction of element $i, G_{i}^{o, \phi}$ is the Gibbs energy of $\phi$ phase of pure element $i$, and ${ }^{x s} G^{\mathrm{bn}, \phi}$ and ${ }^{x s} G^{\mathrm{tern}, \phi}$ are the excess Gibbs energies of the constitutive binary and ternary systems, respectively. The excess Gibbs energies for binary and ternary systems can be further described as

$$
{ }^{x s} G^{\mathrm{bin}}=\sum_{i=1}^{c-1} \sum_{j>i}^{c} x_{i} x_{j} \sum_{v=0}^{n}{ }^{v} L_{i j}^{\phi}\left(x_{i}-x_{j}\right)^{v},
$$




$$
{ }^{x s} G^{\mathrm{tern}}=\sum_{i=1}^{c-2} \sum_{j>i}^{c-1} \sum_{k>j}^{c} x_{i} x_{j} x_{k}\left(L_{i}^{\phi} x_{i}+L_{j}^{\phi} x_{j}+L_{k}^{\phi} x_{k}\right),
$$

where ${ }^{v} L_{i j}^{\phi}$ is the $v$ th order interaction parameter ${ }^{13}$ in a binary and normally described as

$$
{ }^{v} L_{i j}^{\phi}={ }^{v} a+{ }^{v} b T,
$$

where ${ }^{v} a$ and ${ }^{v} b$ are model parameters evaluated from experimental information. The ternary parameter of element $i, L_{i}^{\phi}$, in Eq. (3) also has the form of Eq. (4). If all three $L$ parameters in Eq. (3) are identical, as in a ternary regular solution, ${ }^{6}$

$$
L_{i}^{\phi}=L_{j}^{\phi}=L_{k}^{\phi}=L_{i j k}^{\phi}
$$

then the ternary excess Gibbs energy shown in Eq. (3) can be further simplified to

$$
{ }^{x s} G^{\mathrm{tern}}=\sum_{i=1}^{c-2} \sum_{j>i}^{c-1} \sum_{k>j}^{c} x_{i} x_{j} x_{k} L_{i j k}^{\phi},
$$

since $x_{i}+x_{j}+x_{k}=1$ in a ternary.

\section{TERNARY fcc SPECIAL QUASIRANDOM STRUCTURES}

Thermodynamic properties of solid solution phases can be calculated in several ways (e.g., see Refs. 14-19). A popular approach is to use a database of first-principles calculations to determine the so-called effective cluster interactions that describe the energetics of the alloy system of interest. These interactions are then used as an input for efficient statistical mechanics methods, such as the cluster variation method or Monte Carlo simulations. While this general approach is able to model ordered phases (with potential point defects) and disordered (with potential short-range order) within a unified framework, it can be computationally demanding. Fortunately, in cases where the sole objective is to obtain a reliable thermodynamic model for disordered phases that can be reasonably assumed to lack significant short-range order, the use of SQS provides a considerably more straightforward and computationally efficient approach.

As discussed in Sec. I, first-principles study of SQS can effectively consider the limitations discussed above, and it has been demonstrated that binary SQSs can be applied to calculate thermodynamic properties of binary solid solutions, such as mixing enthalpy, for fcc, bcc, and hcp structures. ${ }^{10-12}$ It is thus anticipated that first-principles calculations of ternary SQSs should be able to reproduce thermodynamic properties of ternary solid solutions if their atomic configurations, which are represented as correlation functions, are very close to those of ternary solid solutions. Correlation functions of solid solution phases are well derived by Inden and Pitsch. ${ }^{20}$ In the following section, the correlation functions for binary and ternary systems are briefly summarized.

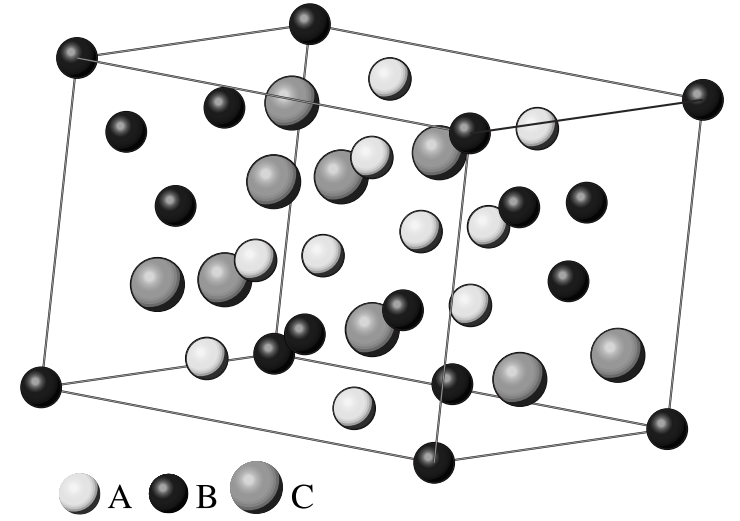

(a) SQS-24 when $x_{A}=x_{B}=x_{C}=\frac{1}{3}$

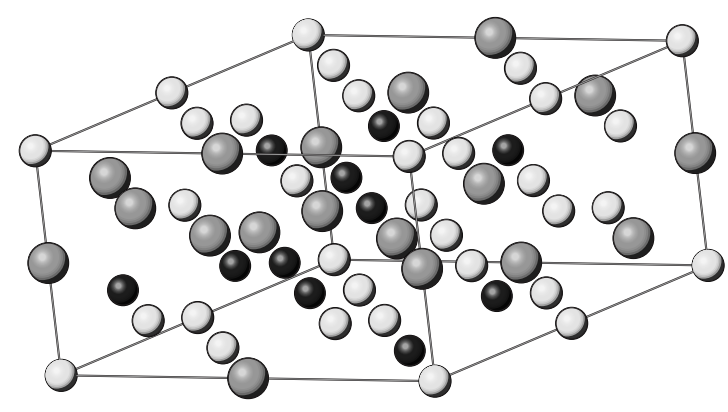

(b) SQS-32 when $x_{A}=\frac{1}{2}, x_{B}=x_{C}=\frac{1}{4}$

FIG. 1. Atomic arrangements of the ternary fcc SQSs in their ideal, unrelaxed forms. All the atoms are at their ideal fcc sites, even though both structures have the space group $P 1$.

\section{A. Correlation functions}

The normalized correlation functions $\bar{\Pi}_{k}$ in crystalline structures are defined as

$$
\bar{\Pi}_{k}=\Pi_{12 \cdots k}^{c_{1} c_{2} \cdots c_{k}}=\frac{1}{N_{k}} \sum_{\text {site }} \sigma_{1}^{c_{1}-1} \sigma_{2}^{c_{2}-1} \cdots \sigma_{k}^{c_{k}-1}
$$

where the sum is over all the distinctive $k$-site clusters, which are geometrically equivalent, in the $N$ lattice site structure. When $k=1,2,3, \ldots$, then $k$-site clusters are point, pair, triplets, and so forth. Site occupation variables are denoted as $\sigma_{k}$, where the subscript $k$ indicates that the $k$ th constituent is located at the corresponding site. The superscript $c_{k}$ takes values of $2,3, \ldots, C$, with $C$ as the number of constituents, which represents a constituent $c_{k}$ at a given lattice site.

For binary systems when $C=2$, conventional values of the site occupation variables $\sigma_{k}$ are \pm 1 depending on whether a lattice site is occupied by $A$ or $B$ atoms. According to Eq. (7), the normalized point correlation function for the second constituent site ( $B$ atom sites) is given as $\Pi_{1}^{2}=\frac{1}{N_{1}} \sum \sigma_{1}^{2-1}$, with $\sigma_{1}=1$ or -1 . It is worth noting that the atom sites do not need to be distinguished in a binary system since they are switchable. With the mole fractions of $A$ and $B$ being $x_{A}$ and $x_{B}$, respectively $\left(x_{A}+x_{B}=1\right)$, then $\Pi_{1}^{2}=x_{A}-x_{B}$. For a $k$-site 
TABLE I. Pair and multisite correlation functions of ternary fcc SQSs when $x_{A}=x_{B}=x_{C}=\frac{1}{3}$. The number in square brackets next to $\bar{\Pi}_{k, m}$ is the number of equivalent figures at the same distance in the structure, the so-called degeneracy factor.

\begin{tabular}{|c|c|c|c|c|c|c|c|c|c|}
\hline & \multirow[b]{2}{*}{ Random } & \multicolumn{8}{|c|}{ SQS- $N$} \\
\hline & & 3 & 6 & 9 & 15 & 18 & 24 & 36 & 48 \\
\hline $\bar{\Pi}_{2,1}[6]$ & 0 & 0 & 0 & 0 & 0 & 0 & 0 & 0 & 0 \\
\hline $\bar{\Pi}_{2,1}[12]$ & 0 & 0 & 0 & 0 & 0 & 0 & 0 & 0 & 0 \\
\hline $\bar{\Pi}_{2,1}[6]$ & 0 & 0 & 0 & 0 & 0 & 0 & 0 & 0 & 0 \\
\hline $\bar{\Pi}_{2,2}[3]$ & 0 & 0.25 & -0.125 & 0 & 0 & 0 & 0 & 0 & 0 \\
\hline $\bar{\Pi}_{2,2}[6]$ & 0 & 0 & 0 & 0 & 0 & 0 & 0 & 0 & 0 \\
\hline $\bar{\Pi}_{2,2}[3]$ & 0 & 0.25 & -0.125 & 0 & 0 & 0 & 0 & 0 & 0 \\
\hline $\bar{\Pi}_{2,3}[12]$ & 0 & -0.25 & -0.0625 & -0.0625 & 0 & -0.01042 & 0 & 0 & 0 \\
\hline $\bar{\Pi}_{2,3}[24]$ & 0 & 0 & 0 & 0 & 0 & 0 & 0 & 0 & 0 \\
\hline $\bar{\Pi}_{2,3}[12]$ & 0 & -0.25 & -0.0625 & -0.0625 & -0.06667 & -0.01042 & 0 & 0 & 0 \\
\hline $\bar{\Pi}_{2,4}[6]$ & 0 & 0 & 0 & 0 & -0.075 & 0 & 0 & -0.04167 & 0 \\
\hline $\bar{\Pi}_{2,4}[12]$ & 0 & 0 & 0 & 0 & -0.01443 & 0.0842 & 0 & 0 & -0.02255 \\
\hline $\bar{\Pi}_{2,4}[6]$ & 0 & 0 & 0 & 0 & -0.05833 & 0.09722 & 0.04167 & -0.04167 & 0.09896 \\
\hline $\bar{\Pi}_{3,1}[8]$ & 0 & 0.125 & -0.01563 & 0.03125 & 0.04063 & 0.03125 & 0.01953 & -0.00391 & 0.01953 \\
\hline $\bar{\Pi}_{3,1}[24]$ & 0 & 0 & 0 & 0 & -0.03789 & 0 & 0.01353 & 0.00226 & 0.00338 \\
\hline $\bar{\Pi}_{3,1}[24]$ & 0 & -0.125 & 0.01563 & -0.03125 & 0.00938 & -0.03125 & -0.00391 & -0.02734 & 0 \\
\hline $\bar{\Pi}_{3,1}[8]$ & 0 & 0 & 0 & 0 & -0.00541 & 0 & 0.01353 & -0.00226 & 0.01691 \\
\hline $\bar{\Pi}_{3,2}[12]$ & 0 & -0.125 & 0.0625 & 0 & -0.025 & 0 & -0.01562 & 0 & -0.00391 \\
\hline $\bar{\Pi}_{3,2}[24]$ & 0 & 0 & 0 & 0 & 0.2165 & 0.01804 & 0 & 0.00902 & 0 \\
\hline $\bar{\Pi}_{3,2}[12]$ & 0 & 0 & 0 & 0 & 0 & -0.03608 & 0.02706 & -0.01804 & 0.00677 \\
\hline $\bar{\Pi}_{3,2}[24]$ & 0 & 0.125 & -0.0625 & 0 & -0.0125 & 0.01042 & 0.01563 & 0.01563 & -0.00781 \\
\hline $\bar{\Pi}_{3,2}[12]$ & 0 & 0.125 & -0.0625 & 0 & -0.025 & -0.02083 & -0.01562 & -0.01042 & -0.02734 \\
\hline $\bar{\Pi}_{3,2}[12]$ & 0 & 0 & 0 & 0 & 0 & 0 & 0.00902 & 0 & 0.00226 \\
\hline
\end{tabular}

cluster, the normalized correlation functions for the binary solid solution are formulated as

$$
\bar{\Pi}_{k}=\left(x_{A}-x_{B}\right)^{k} .
$$

Similarly, for ternary systems when $C=3$, the values of the site occupation variables $\sigma_{k}$ conventionally take $+1,0$, or -1 if a lattice site is occupied by $A, B$, or $C$ atoms, respectively. The normalized point correlation function for the second constituent site ( $B$ atom sites) is given as $\Pi_{1}^{2}=\frac{1}{N_{1}} \sum \sigma_{1}^{2-1}$, with $\sigma_{1}=+1,0$, or, -1 . For the third constituent site ( $C$ atom sites), the correlation function can be given as $\Pi_{1}^{3}$ $=\frac{1}{N_{1}} \sum \sigma_{1}^{3-1}$, with $\sigma_{1}=+1,0$, or, -1 . With the mole fractions of $A, B$, and $C$ being $x_{A}, x_{B}$, and $x_{C}$, respectively $\left(x_{A}+x_{B}\right.$ $\left.+x_{C}=1\right)$, then $\Pi_{1}^{2}=x_{A}-x_{C}$ and $\Pi_{1}^{3}=x_{A}+x_{C}$. The vanishing of $x_{B}$ is due to its site occupation variable being 0 . For a $k$-site cluster with $n_{B} B$ atom sites and $n_{C} C$ atom sites $\left(n_{B}+n_{C}\right.$ $=k$ ), the normalized correlation functions for the ternary solid solution are denoted as

$$
\bar{\Pi}_{k}=\left(x_{A}-x_{C}\right)^{n_{B}}\left(x_{A}+x_{C}\right)^{n_{C}} \quad \text { with } n_{B}+n_{C}=k .
$$

\section{B. Generation of ternary fcc special quasirandom structure}

In the present work, two different ternary fcc SQSs are generated. The first SQS is at the equimolar composition where $x_{A}=x_{B}=x_{C}=\frac{1}{3}$ and the second is at $x_{A}=\frac{1}{2}, x_{B}=x_{C}=\frac{1}{4}$. By switching the occupation of the $A$ atoms in the second SQS with either $B$ or $C$ atoms, two other SQSs can be obtained where $x_{A}=\frac{1}{4}, x_{B}=\frac{1}{2}, x_{C}=\frac{1}{4}$ and $x_{A}=x_{B}=\frac{1}{4}, x_{C}=\frac{1}{2}$. Therefore, mixing enthalpy at four different compositions in a ternary system can be determined from first-principles total energy calculations of ternary fcc SQSs by

$$
\Delta H\left(A_{a} B_{b} C_{c}\right) \approx E\left(A_{a} B_{b} C_{c}\right)-x_{A} E(A)-x_{B} E(B)-x_{C} E(C),
$$

where $E$ represents the total energy of each structure, and the reference states for all pure elements are fcc.

When the number of atoms in the ternary SQS is less than 24 , the alloy theoretic automated toolkit ${ }^{21}$ (ATAT) has been used to generate ternary fcc SQSs. Since the ATAT enumerates all the atomic configurations within each supercell and then checks its correlation functions, the time needed to find SQSs increases exponentially as the size of a supercell in- 
TABLE II. Pair and multisite correlation functions of ternary fcc SQSs when $x_{A}=\frac{1}{2}, x_{B}=x_{C}=\frac{1}{4}$. The number in square brackets next to $\bar{\Pi}_{k, m}$ is the number of equivalent figures at the same distance in the structure, the so-called degeneracy factor.

\begin{tabular}{|c|c|c|c|c|c|c|c|c|}
\hline & \multirow[b]{2}{*}{ Random } & \multicolumn{7}{|c|}{ SQS-N } \\
\hline & & 4 & 8 & 16 & 24 & 32 & 48 & 64 \\
\hline $\bar{\Pi}_{2,1}[6]$ & 0.0625 & 0.0625 & 0.0625 & 0.0625 & 0.0625 & 0.0625 & 0.0625 & 0.0625 \\
\hline $\bar{\Pi}_{2,1}[12]$ & 0 & 0 & 0 & 0 & 0 & 0 & 0 & 0 \\
\hline $\bar{\Pi}_{2,1}[6]$ & 0 & -0.0625 & 0 & 0 & 0 & 0 & 0 & 0 \\
\hline $\bar{\Pi}_{2,2}[3]$ & 0.0625 & -0.125 & 0.0625 & 0.0625 & -0.0625 & 0.0625 & 0.0625 & 0.0625 \\
\hline $\bar{\Pi}_{2,2}[6]$ & 0 & 0 & 0 & 0 & 0.09021 & 0 & 0 & 0 \\
\hline $\bar{\Pi}_{2,2}[3]$ & 0 & 0.125 & 0.0625 & -0.0625 & 0.08333 & 0 & 0 & 0 \\
\hline $\bar{\Pi}_{2,3}[12]$ & 0.0625 & 0.0625 & -0.00781 & 0.074219 & 0.0625 & 0.0625 & 0.0625 & 0.05957 \\
\hline $\bar{\Pi}_{2,3}[24]$ & 0 & 0 & 0 & 0.006766 & 0.02255 & 0 & -0.002255 & 0.003383 \\
\hline $\bar{\Pi}_{2,3}[12]$ & 0 & -0.0625 & -0.10156 & 0.011719 & 0.02604 & 0 & -0.002604 & -0.006836 \\
\hline $\bar{\Pi}_{2,4}[6]$ & 0.0625 & -0.125 & 0.015625 & 0.085938 & 0.15625 & 0.0625 & 0.0625 & 0.361328 \\
\hline $\bar{\Pi}_{2,4}[12]$ & 0 & 0 & 0 & -0.040595 & 0 & -0.05413 & 0.009021 & -0.010149 \\
\hline $\bar{\Pi}_{2,4}[6]$ & 0 & 0.125 & -0.046875 & -0.023437 & 0.07292 & 0 & -0.03125 & 0.193359 \\
\hline $\bar{\Pi}_{3,1}[8]$ & -0.015625 & -0.015625 & 0.089844 & -0.068359 & -0.015625 & -0.05518 & 0.001953 & -0.015625 \\
\hline $\bar{\Pi}_{3,1}[24]$ & 0 & 0 & 0 & 0.010149 & -0.013532 & 0.00254 & -0.003383 & 0.010149 \\
\hline $\bar{\Pi}_{3,1}[24]$ & 0 & 0.015625 & -0.058594 & 0.005859 & -0.015625 & 0.01025 & -0.005859 & 0.008789 \\
\hline $\bar{\Pi}_{3,1}[8]$ & 0 & 0 & 0 & -0.010149 & 0.013532 & 0.00761 & 0.02368 & 0.005074 \\
\hline $\bar{\Pi}_{3,2}[12]$ & -0.015625 & 0.03125 & -0.015625 & 0.019531 & 0.015625 & -0.05078 & -0.033203 & -0.050781 \\
\hline $\bar{\Pi}_{3,2}(24)$ & 0 & 0 & 0 & 0.010149 & -0.036084 & -0.01015 & 0 & -0.025372 \\
\hline $\bar{\Pi}_{3,2}[12]$ & 0 & 0 & 0 & -0.040595 & -0.027063 & -0.02030 & -0.003383 & 0.030446 \\
\hline $\bar{\Pi}_{3,2}[24]$ & 0 & -0.03125 & 0.046875 & 0.005859 & 0.007813 & 0 & 0.003906 & -0.008789 \\
\hline $\bar{\Pi}_{3,2}[12]$ & 0 & -0.03125 & 0.078125 & 0.007813 & -0.036458 & -0.01172 & 0.013672 & -0.017578 \\
\hline $\bar{\Pi}_{3,2}[12]$ & 0 & 0 & 0 & 0.006766 & 0 & 0 & 0.001128 & -0.020297 \\
\hline
\end{tabular}

creases. For the sake of efficiency, to find SQSs bigger than 24 lattice sites, a Monte Carlo-like scheme ${ }^{22}$ has been used. In each supercell with different lattice vectors, atom positions are randomly exchanged between the atoms, and correlation functions of a supercell are calculated after every alternation. If the correlation functions of a new state are getting closer to those of random solutions, then the new configuration is accepted. Otherwise the new state is discarded and another configuration will be generated from the previous one. This process continues until the atomic arrangement of a supercell converges to the closest correlation functions of the random solution. In both methods, direct search via ATAT and Monte Carlo-like scheme, a supercell whose correlation functions match best with that of a completely random structure is chosen as the SQS at a given number of lattice sites.

The selected SQSs at two different compositions, SQS-24 when $x_{A}=x_{B}=x_{C}=\frac{1}{3}$ and SQS-32 when $x_{A}=\frac{1}{2}$ and $x_{B}=x_{C}=\frac{1}{4}$, are shown in Fig. 1. These two SQSs are selected for later calculations because they are adequate with respect to their size and correlation functions at each concentration. ${ }^{31}$ The space groups of both structures are $P 1$ with all the atoms at their ideal fcc sites. The correlation functions of the generated two SQSs are given in Tables I and II, respectively.

\section{FIRST-PRINCIPLES METHODOLOGY}

The Vienna $a b$ initio simulation package ${ }^{23}$ (VASP) was used to perform the density functional theory (DFT) electronic structure calculations. The projector augmented wave method $^{24}$ was chosen and the generalized gradient approximation $^{25}$ was used to take into account exchange and correlation contributions to the Hamiltonian of the ionelectron system. An energy cutoff of $364 \mathrm{eV}$ was used to calculate the electronic structures of all the SQSs. $5000 k$ points per reciprocal atom based on the Monkhorst-Pack scheme for the Brillouin-zone sampling were used.

In all first-principles calculations of binary and ternary SQSs in the present work, structures are relaxed in two ways: full relaxation and volume relaxation to preserve the fcc symmetry. It should be emphasized here that the preserved symmetry is that of SQS when all the atoms are substituted into a single element, which is the underlying fcc symmetry. For the full relaxation of SQS, structures are completely re- 


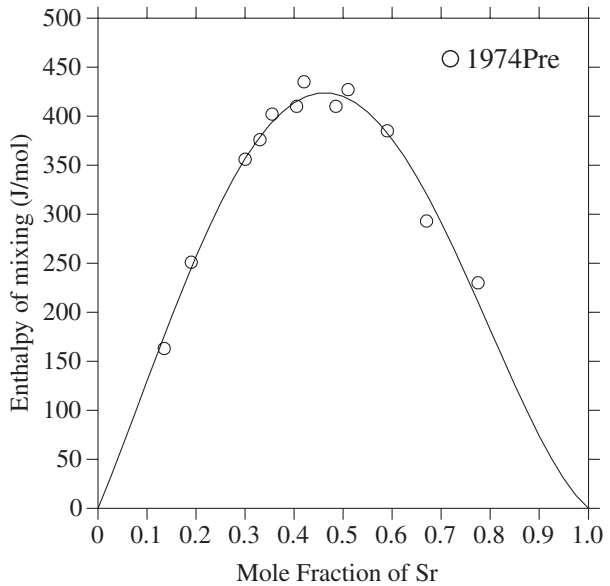

(a) $\mathrm{Ca}-\mathrm{Sr}$ (liquid)

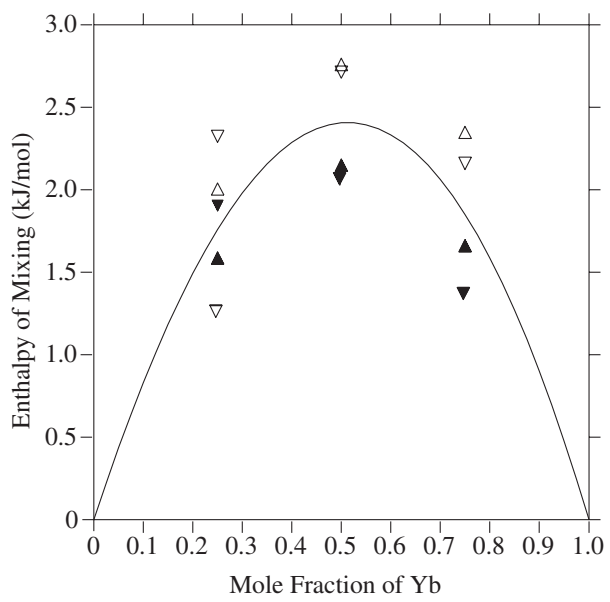

(c) $\mathrm{Sr}-\mathrm{Yb}(\mathrm{fcc})$

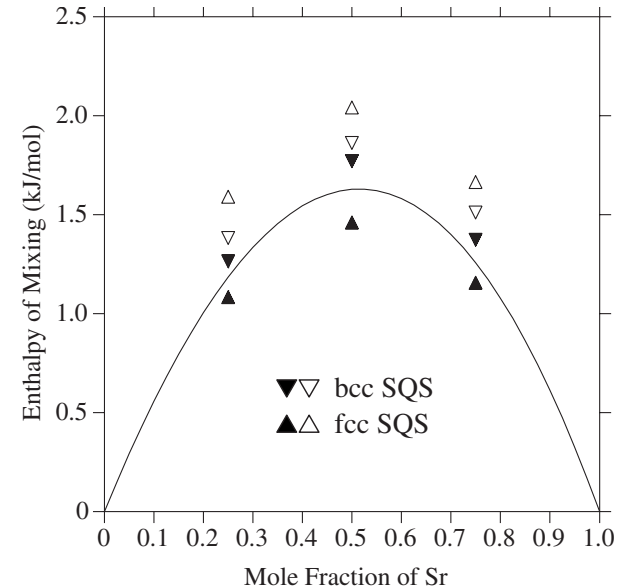

(b) $\mathrm{Ca}-\mathrm{Sr}$ (fcc)

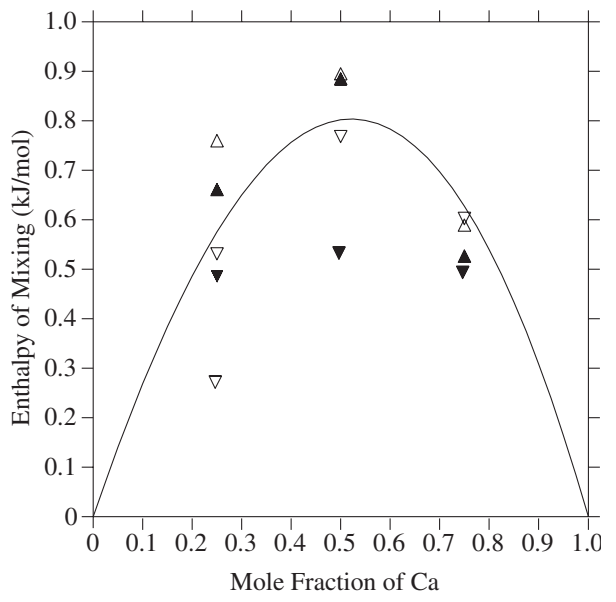

(d) $\mathrm{Yb}-\mathrm{Ca}(\mathrm{fcc})$
FIG. 2. Calculated mixing enthalpies for (a) the liquid phase in the $\mathrm{Ca}-\mathrm{Sr}$ with experimental measurement (Ref. 29) and the fcc phase for (b) Ca-Sr, (c) Sr-Yb, and (d) $\mathrm{Yb}-\mathrm{Ca}$ with first-principles calculations of fully relaxed SQSs. Note that fcc and bcc phases are evaluated with same model parameters. Open and closed symbols represent symmetry-preserved and fully relaxed calculations, respectively. laxed with respect to all degrees of freedom, i.e., cell shape, volume, and ionic positions, while the symmetry-preserving relaxation only allows us to change the cell volume for cubic structures, such as fcc and bcc. In calculating hep SQSs, however, relaxing only the volume will fix the $c / a$ ratio of the underlying hcp symmetry. ${ }^{12}$ Therefore, the shape of SQSs has to be relaxed as well as the volume in hcp SQS calculations. Since the present work focuses on the calculations of fcc SQSs, symmetry-preserving relaxation is equivalent to volume relaxation. For symmetry-preserved calculations, all the atoms are still at the lattice sites of fcc's and only the effective lattice parameter of fcc changes with this constrained relaxation scheme. However, the local relaxation due to the like and dislike bondings cannot be taken into account. Further discussion with regard to the different relaxation schemes of SQSs can be found in Ref. 12 .

\section{RESULTS AND DISCUSSIONS}

The mixing enthalpy derived from first-principles calculations of ternary fcc SQSs should be compared to relevant experimental measurements. However, there are always ordering effects at low temperatures where the fcc solid solutions show complete solubility in binaries, such as $\mathrm{Cu}-\mathrm{Au}$ and Au-Pd systems. Due to such ordering in binaries, it is almost impossible to reliably determine the mixing enthalpy for ternary fcc solid solutions from experiments.

In this work, the $\mathrm{Ca}-\mathrm{Sr}-\mathrm{Yb}$ system has been selected to apply the generated ternary fcc SQSs, which presumably has complete solubility in the fcc phase for all binaries and ternary without any reported order-disorder transition. Both the $\mathrm{Ca}-\mathrm{Sr}$ and $\mathrm{Ca}-\mathrm{Yb}$ systems show complete solubility for both fcc and bcc phases at low and high temperatures, respectively, without the formation of any intermetallic compounds. ${ }^{26,27}$ There is no reported phase diagram for the $\mathrm{Sr}-\mathrm{Yb}$ system; however, from the similarity of the two binary systems, $\mathrm{Ca}-\mathrm{Sr}$ and $\mathrm{Ca}-\mathrm{Yb}$, it can be postulated that $\mathrm{Sr}-\mathrm{Yb}$ would also have complete solubility for both fcc and bcc phases. Consequently, it may cautiously be expected that the combined ternary, the Ca-Sr-Yb system, would have the fcc solid solution phase throughout the entire composition range at low temperatures.

\section{A. Binary special quasirandom structures for the Ca-Sr-Yb system}

Prior to applying the ternary SQSs to the $\mathrm{Ca}-\mathrm{Sr}-\mathrm{Yb}$ system, the mixing behavior of the fcc phase in its constitutive three binaries was investigated through eight-atom binary fcc SQSs at three different compositions, namely, $x=0.25,0.5$, 


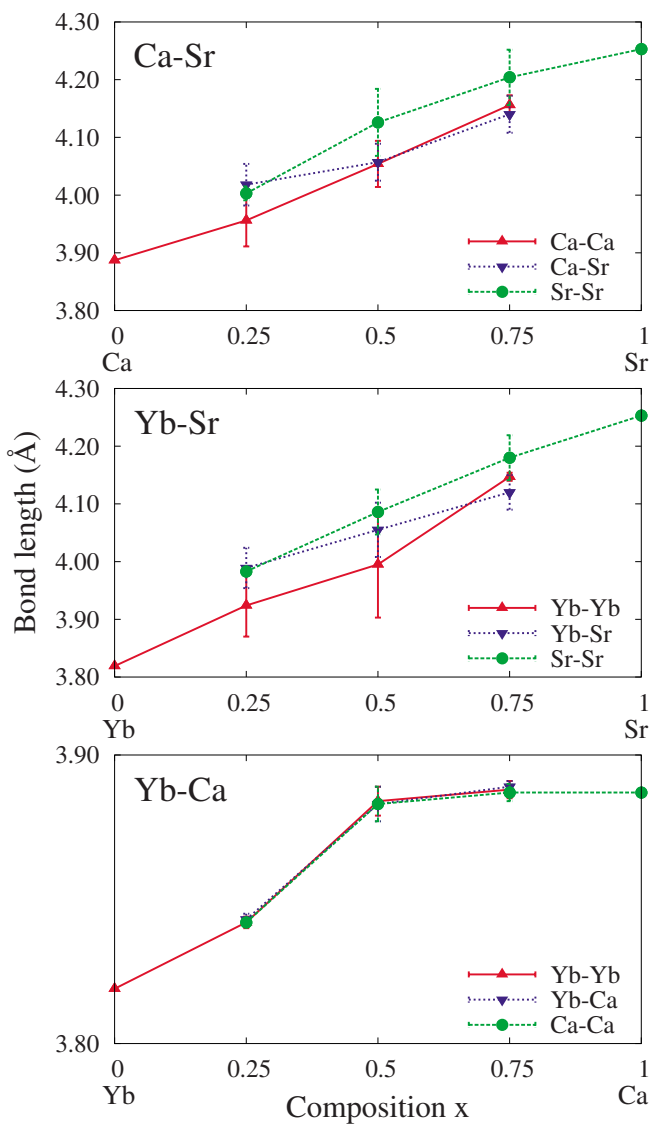

FIG. 3. (Color online) SQS bond lengths for three binaries, Ca$\mathrm{Sr}, \mathrm{Yb}-\mathrm{Sr}$, and $\mathrm{Yb}-\mathrm{Ca}$. Error bars correspond to the standard deviation of the bond length distributions.

and 0.75 in $A_{1-x} B_{x}$ alloys. Calculated mixing enthalpies from binary fcc SQSs are combined with experimental data from the literature ${ }^{28,29}$ to evaluate interaction parameters in Eq. (4) for each binary. For the sake of simplicity, parameters for bcc have been modeled as identical to those of fcc. Binary bcc SQSs have also been calculated and included in the parameter evaluation process. Figure 2 shows that the formation energies of binary bcc and fcc SQSs are very close to each other in all cases. The congruent melting of bcc is observed in both the $\mathrm{Ca}-\mathrm{Sr}$ and $\mathrm{Yb}-\mathrm{Ca}$ systems; thus, the $\mathrm{Sr}-\mathrm{Yb}$ system has been modeled to have it as well on the assumption that $\mathrm{Sr}-\mathrm{Yb}$ would behave similarly.

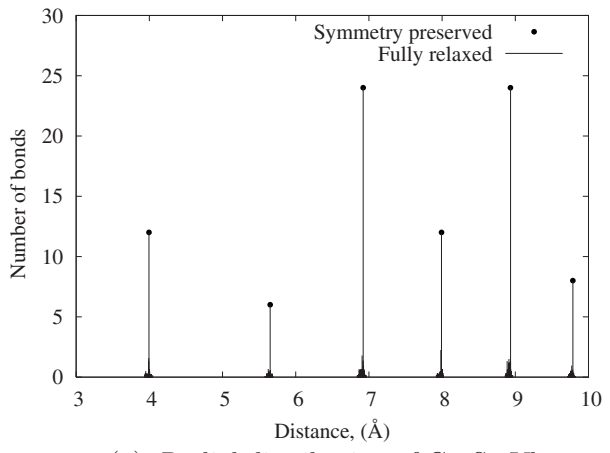

(a) Radial distribution of $\mathrm{Ca}_{1} \mathrm{Sr}_{1} \mathrm{Yb}_{1}$

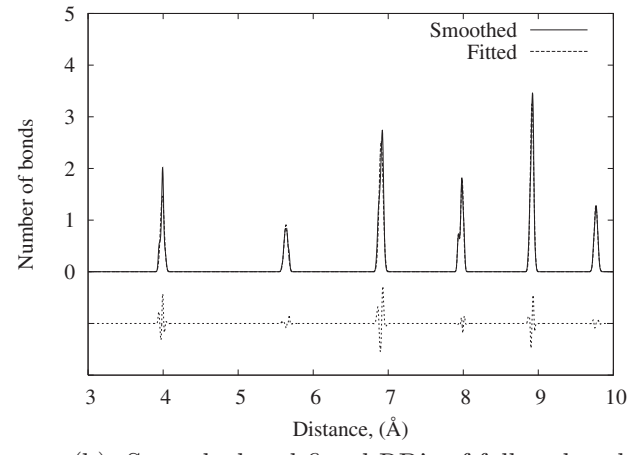

(b) Smoothed and fitted RD's of fully relaxed $\mathrm{Ca}_{1} \mathrm{Sr}_{1} \mathrm{Yb}_{1}$
FIG. 5. Radial distribution analysis of $\mathrm{Ca}_{1} \mathrm{Sr}_{1} \mathrm{Yb}_{1}$ ternary fcc SQSs. The dotted line in (b) under the smoothed and fitted curves is the error between the two curves. 


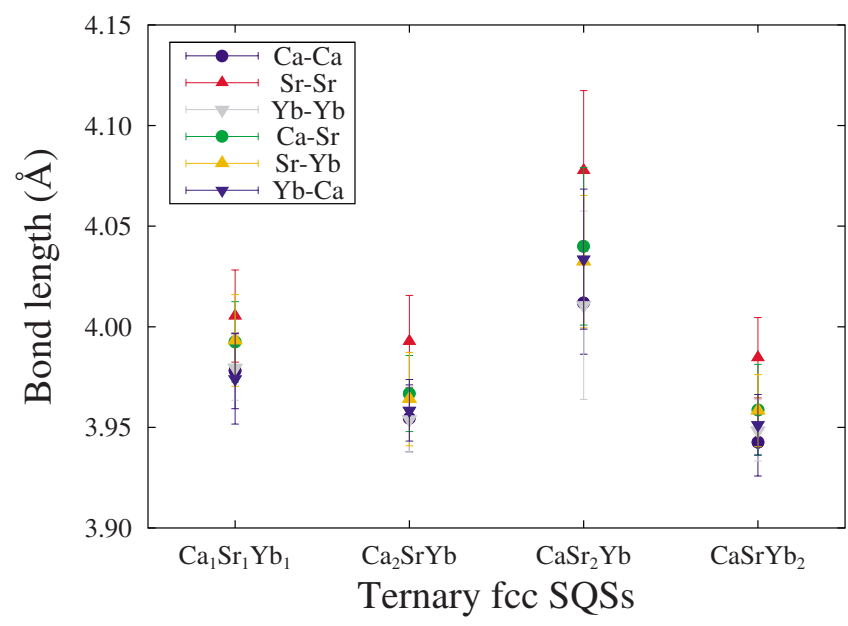

FIG. 6. (Color online) First nearest-neighbor average bond lengths for the fully relaxed ternary fcc SQSs in the Ca-Sr-Yb system. Error bar corresponds to the standard deviation of the bond length distributions.

SQS calculations shows that the local relaxation effect in both fcc and bcc SQSs is small after the full relaxation, and the difference between fully relaxed and symmetry-preserved structures is at most $\sim 1 \mathrm{~kJ} / \mathrm{mol}$.

It is intriguing to see that only the $\mathrm{Yb}-\mathrm{Ca}$ system has the mixing enthalpy close to zero among three binaries in Fig. 2, which implies that $\mathrm{Yb}-\mathrm{Ca}$ is likely to have ideal mixing in both fcc and bcc phases. The ideal mixing of $\mathrm{Yb}$ and $\mathrm{Ca}$ is attributed to their similar lattice parameters. The other two systems, $\mathrm{Ca}-\mathrm{Sr}$ and $\mathrm{Sr}-\mathrm{Yb}$, have rather positive $(\sim 2 \mathrm{~kJ} / \mathrm{mol})$ mixing enthalpies and significant lattice parameter differences $\left(a_{\mathrm{Ca}} \sim a_{\mathrm{Yb}} \ll a_{\mathrm{Sr}}\right)$, which can be the reason for the nonideal mixing behavior, unlike $\mathrm{Yb}-\mathrm{Ca}$.

The bond length analysis for the fully relaxed SQSs in Fig. 3 shows that first nearest-neighbor average bond lengths follow Vegard's law closely in all calculations. This observation indicates that the lattice parameter of the fcc solid solution varies linearly with the composition change and there is no significant geometrical distortion.

\section{B. Ternary fec special quasirandom structures for the Ca-Sr- Yb system}

First-principles calculations of ternary fcc SQSs at four different compositions in the $\mathrm{Ca}-\mathrm{Sr}-\mathrm{Yb}$ system (namely, $x_{\mathrm{Ca}}$ $=x_{\mathrm{Sr}}=x_{\mathrm{Yb}}=\frac{1}{3} ; x_{\mathrm{Ca}}=\frac{1}{2}, x_{\mathrm{Sr}}=x_{\mathrm{Yb}}=\frac{1}{4} ; x_{\mathrm{Sr}}=\frac{1}{2}, x_{\mathrm{Ca}}=x_{\mathrm{Yb}}=\frac{1}{4}$; and $x_{\mathrm{Yb}}=\frac{1}{2}, x_{\mathrm{Ca}}=x_{\mathrm{Sr}}=\frac{1}{4}$ ) have been considered to investigate the ternary interactions. Three isoplethal sections, connecting the equimolar composition and three other compositions when $x_{i}=1 / 2, x_{j} / x_{k}=1$, are selected to examine the mixing enthalpy for the $\mathrm{Ca}-\mathrm{Sr}-\mathrm{Yb}$ ternary system.

Calculated enthalpies of mixing from ternary fcc SQSs are shown in Fig. 4, including extrapolated results from the three binaries and improved enthalpies of mixing to reproduce ternary fcc SQS results by introducing ternary interaction parameters. All the fully relaxed ternary SQSs show that the effect of local relaxation is also small as in its constitutive binaries; thus, the energy differences between the symmetry-preserved and fully relaxed calculations are quite small. Figure 5 shows the radial distribution analysis of the fully relaxed SQS at the equimolar composition. The narrow distribution along each of the bond lengths indicates that the effect of local relaxation is small. As shown in Fig. 4, energy differences between fully relaxed and symmetry-preserved calculations are small $(\sim 0.5 \mathrm{~kJ} / \mathrm{mol})$.

As can be seen in Fig. 4, the mixing enthalpy extrapolated from the three binaries is slightly lower than that derived from first-principles calculations of ternary fcc SQSs. Thus, ternary interaction parameters are introduced to improve the ternary mixing enthalpy. According to Eq. (3), the contribu-

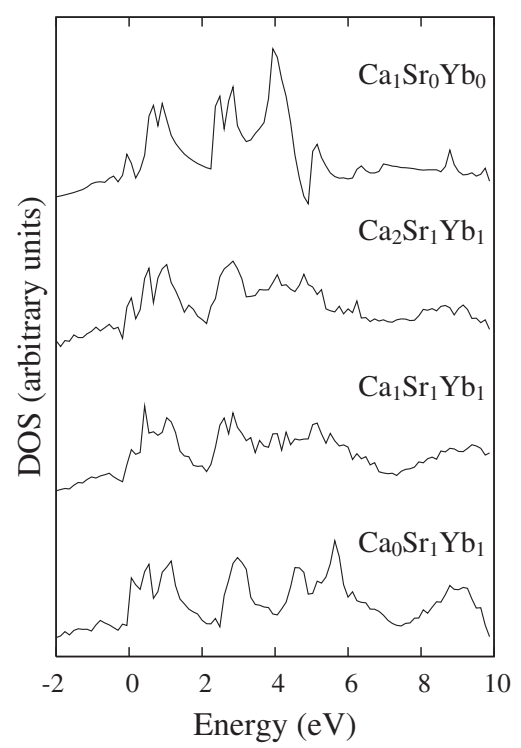

(a) $\mathrm{Ca}-\mathrm{SrYb}$

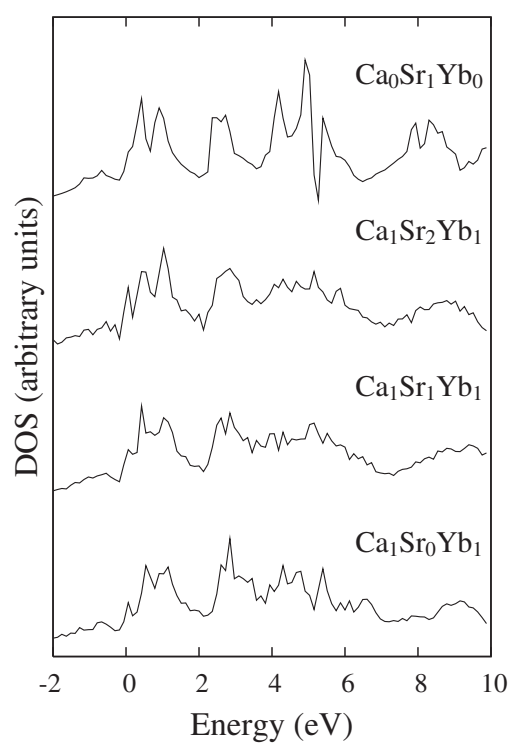

(b) $\mathrm{Sr}-\mathrm{YbCa}$

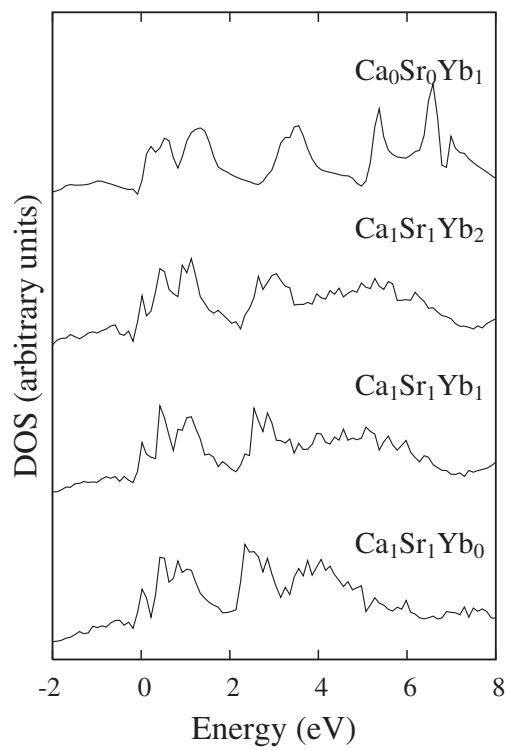

(c) $\mathrm{Yb}-\mathrm{CaSr}$

FIG. 7. Calculated density of states for $\mathrm{Ca}-\mathrm{Sr}-\mathrm{Yb}$ as three pseudobinaries. 
tion from the ternary excess Gibbs energy for the fcc phase in the $\mathrm{Ca}-\mathrm{Sr}-\mathrm{Yb}$ system can be denoted as

$$
{ }^{x s} G^{\mathrm{tern}, \mathrm{fcc}}=x_{\mathrm{Ca}} x_{\mathrm{Sr}} x_{\mathrm{Yb}}\left(L_{\mathrm{Ca}}^{\mathrm{fcc}} x_{\mathrm{Ca}}+L_{\mathrm{Sr}}^{\mathrm{fcc}} x_{\mathrm{Sr}}+L_{\mathrm{Yb}}^{\mathrm{fcc}} x_{\mathrm{Yb}}\right)
$$

or

$$
{ }^{x s} G^{\mathrm{tern}, \mathrm{fcc}}=x_{\mathrm{Ca}} x_{\mathrm{Sr}} x_{\mathrm{Yb}} L_{\mathrm{CaSrYb}}^{\mathrm{fcc}},
$$

as simplified in Eq. (6) when the ternary fcc is considered as a regular solution. When three independent ternary interaction parameters $\left(L_{\mathrm{Ca}}=25940 \mathrm{~J} / \mathrm{mol}, L_{\mathrm{Sr}}=2913 \mathrm{~J} / \mathrm{mol}\right.$, and $L_{\mathrm{Yb}}=-8645 \mathrm{~J} / \mathrm{mol}$ ) are used, slightly better agreement with ternary SQSs was made than with a single regular interaction parameter $\left(L_{\mathrm{CaSrYb}}=6736 \mathrm{~J} / \mathrm{mol}\right)$. The calculated mixing enthalpy at the equimolar composition is evaluated as the same value regardless of the interaction parameters since all the data are equally weighted.

Six different bond lengths, three like bondings and three dislike bondings, of four fully relaxed SQSs have been analyzed. In Fig. 6, the bond lengths corresponding to the first nearest neighbors for all SQSs are presented. In all SQS calculations, the Sr-Sr bonding is always the longest, and this is attributed to the biggest lattice parameter of $\mathrm{Sr}$ among the three elements.

As a final analysis of the ability of the generated SQSs to reproduce the properties of ternary fcc solid solutions, Fig. 7 shows the alloying effects on the electronic density of states (DOS) in the Ca-Sr-Yb system. Three pseudobinaries, connecting a pure element and equimolar composition of the other two elements, have been selected. Since all three elements have the same number of valence electrons, significant changes in the electronic DOS were not observed. Instead, the electronic DOS of the outer states gradually transformed into that of the pure element as the composition changed toward the pure element. The peak around $\sim 9 \mathrm{eV}$ of $\mathrm{SrYb}$ in Fig. 7(a) flattens out as $\mathrm{Ca}$ increases, and the peaks around $\sim 8$ and $\sim 7 \mathrm{eV}$ become more pronounced as the content of $\mathrm{Sr}$ and $\mathrm{Yb}$ increases as shown in Figs. 7(b) and 7(c), respectively.

\section{CONCLUSION}

In the present work, two ternary fcc SQSs at different compositions, $x_{A}=x_{B}=x_{C}=\frac{1}{3}$ and $x_{A}=\frac{1}{2}, x_{B}=x_{C}=\frac{1}{4}$, are generated and their correlation functions are satisfactorily close to those of random fcc solid solutions. The generated SQSs are applied to the $\mathrm{Ca}-\mathrm{Sr}-\mathrm{Yb}$ system, which presumably has a complete solubility range without order-disorder transitions in ternary fcc solid solutions. Mixing enthalpies for the fcc phase in three binaries are evaluated from first-principles calculations of fcc and bcc SQSs and available experimental data with the CALPHAD approach. It is found that the local relaxation effect of fcc and bcc phases is very small, and mixing enthalpies are slightly positive in all cases. Evaluated mixing enthalpies for the fcc phase in three binaries are then extrapolated to the ternary system.

First-principles results of four ternary SQSs at $x_{\mathrm{Ca}}=x_{\mathrm{Sr}}$ $=x_{\mathrm{Yb}}=\frac{1}{3} ; \quad x_{\mathrm{Ca}}=\frac{1}{2}, \quad x_{\mathrm{Sr}}=x_{\mathrm{Yb}}=\frac{1}{4} ; \quad x_{\mathrm{Sr}}=\frac{1}{2}, \quad x_{\mathrm{Ca}}=x_{\mathrm{Yb}}=\frac{1}{4} ; \quad$ and
$x_{\mathrm{Yb}}=\frac{1}{2}, x_{\mathrm{Ca}}=x_{\mathrm{Sr}}=\frac{1}{4}$ show that the local relaxation effect is also very small in the ternary system. Extrapolated mixing enthalpy from its constitutive binaries is slightly lower than those from first-principles calculations of ternary fcc SQSs. Thus, ternary interaction parameters for fcc solid solution phases are introduced to further improve the ternary mixing enthalpy. It can be concluded that the generated ternary fcc SQSs are able to reproduce thermodynamic properties of ternary fcc solid solutions and can readily be applied to other systems.

\section{ACKNOWLEDGMENTS}

This work is funded by the National Science Foundation (NSF) through Grant No. DMR-0205232. First-principles calculations were carried out on the LION clusters at the Pennsylvania State University supported in part by the NSF Grants No. (DMR-9983532, No. DMR-0122638, and No. DMR-0205232) and in part by the Materials Simulation Center and the Graduate Education and Research Services at the Pennsylvania State University.

\section{APPENDIX A: EVALUATED INTERACTION PARAMETERS}

Interaction parameters for the liquid and solid solution phases (fcc and bcc) in the three binaries of the $\mathrm{Ca}-\mathrm{Sr}-\mathrm{Yb}$ system - as evaluated from the CALPHAD modeling in the present work-are listed in Table III. Notations are explained in Eq. (2).

\section{APPENDIX B: SPECIAL QUASIRANDOM STRUCTURES FOR THE TERNARY fcc SOLUTION PHASE}

Special quasirandom structures are $N$-atom per cell periodic structures designed to have correlation functions close to those of completely random alloys. The ternary fcc SQSs used in this work are presented in Table IV. Lattice vectors are given as $\mathbf{a}, \mathbf{b}$, and $\mathbf{c}$, and atom positions of $A, B$, and $C$ atoms are given as $A_{i}, B_{i}$, and $C_{i}$, respectively.

TABLE III. Evaluated binary interaction parameters for the Ca$\mathrm{Sr}-\mathrm{Yb}$ system (all in SI units). Gibbs energies for pure elements are from the SGTE pure element database (Ref. 30).

\begin{tabular}{lll}
\hline \hline Systems & Phases & \multicolumn{1}{c}{ Evaluated parameters } \\
\hline Ca-Sr & Liquid & $\begin{array}{l}{ }^{0} L=1680+2.68 T,{ }^{1} L=388-1.153 T,{ }^{2} L=-856 \\
+0.631 T\end{array}$ \\
& fcc,bcc & ${ }^{0} L=6511,{ }^{1} L=-382$ \\
Sr-Yb & Liquid & ${ }^{0} L=8850$ \\
& fcc,bcc & ${ }^{0} L=9624,{ }^{1} L=-488$ \\
Yb-Ca & Liquid & ${ }^{0} L=2689,{ }^{1} L=676$ \\
& fcc,bcc & ${ }^{0} L=3207,{ }^{1} L=280$ \\
\hline \hline
\end{tabular}


TABLE IV. Structural descriptions of the SQSs for the ternary fec solid solution. Lattice vectors and atomic positions are given in fractional coordinates of the fcc lattice. Atomic positions are given for the ideal, unrelaxed fcc sites.

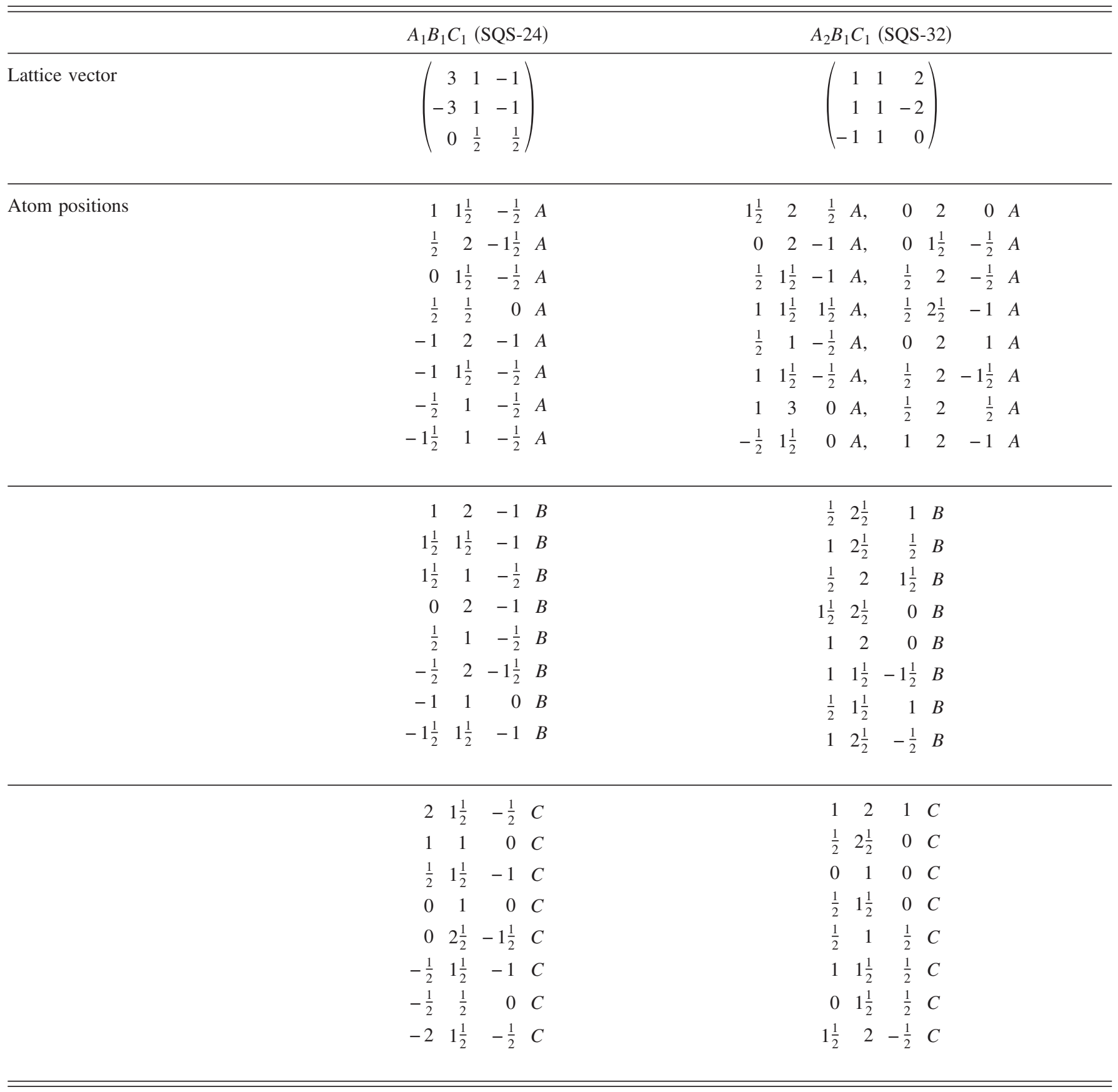

*dus136@psu.edu

${ }^{1}$ L. Kaufman and H. Bernstein, Computer Calculation of Phase Diagram (Academic, New York, 1970).

${ }^{2}$ N. Saunders and A. P. Miodownik, CALPHAD (Calculation of Phase Diagrams): A Comprehensive Guide (Pergamon, Oxford, 1998).

${ }^{3}$ F. Kohler, Monatsch. Chem. 91, 738 (1960).

${ }^{4}$ G. W. Toop, Trans. Soc. Min. Eng. AIME 233, 850 (1965).
${ }^{5}$ Y. M. Muggianu, M. Gambino, and J. P. Bros, J. Chim. Phys. Phys.-Chim. Biol. 72, 83 (1975).

${ }^{6}$ M. Hillert, CALPHAD: Comput. Coupling Phase Diagrams Thermochem. 4, 1 (1980).

${ }^{7}$ K. C. Chou, CALPHAD: Comput. Coupling Phase Diagrams Thermochem. 19, 315 (1995).

${ }^{8}$ Z. Fang and Q. Zhang, J. Chem. Thermodyn. 38, 1079 (2006).

${ }^{9}$ A. Zunger, S. H. Wei, L. G. Ferreira, and J. E. Bernard, Phys. 
Rev. Lett. 65, 353 (1990).

${ }^{10}$ S. H. Wei, L. G. Ferreira, J. E. Bernard, and A. Zunger, Phys. Rev. B 42, 9622 (1990).

${ }^{11}$ C. Jiang, C. Wolverton, J. Sofo, L.-Q. Chen, and Z.-K. Liu, Phys. Rev. B 69, 214202 (2004).

${ }^{12}$ D. Shin, R. Arróyave, Z.-K. Liu, and A. Van de Walle, Phys. Rev. B 74, 024204 (2006).

${ }^{13}$ O. Redlich and A. T. Kister, Ind. Eng. Chem. 40, 345 (1948).

${ }^{14}$ F. Ducastelle, Order and Phase Stability in Alloys, Cohesion and Structure Vol. 3 (Elsevier Science, New York, 1991).

${ }^{15}$ D. de Fontaine, Solid State Physics: Advances in Research and Applications (Academic, New York, 1994), Vol. 47, pp. 33-176.

${ }^{16}$ G. Ceder, A. Van der Ven, C. Marianetti, and D. Morgan, Modell. Simul. Mater. Sci. Eng. 8, 311 (2000).

${ }^{17}$ M. Asta, V. Ozoliṇš, and C. Woodward, JOM 53, 16 (2001).

${ }^{18}$ A. van de Walle and G. Ceder, J. Phase Equilib. 23, 348 (2002).

${ }^{19}$ C. Colinet, in CALPHAD and Alloy Thermodynamics, edited by $\mathrm{P}$. E. A. Turchi, A. Gonis, and R. D. Shull (TMS, Warrendale, PA, 2002), p. 21.

${ }^{20} \mathrm{G}$. Inden and W. Pitsch, Materials Science and Technology: A Comprehensive Treatment (VCH, Weinheim, 1991), Vol. 5, pp.
497-552.

${ }^{21}$ A. van de Walle, M. Asta, and G. Ceder, CALPHAD: Comput. Coupling Phase Diagrams Thermochem. 26, 539 (2002).

${ }^{22}$ I. A. Abrikosov, S. I. Simak, B. Johansson, A. V. Ruban, and H. L. Skriver, Phys. Rev. B 56, 9319 (1997).

${ }^{23}$ G. Kresse and J. Furthmuller, Comput. Mater. Sci. 6, 15 (1996).

${ }^{24}$ G. Kresse and D. Joubert, Phys. Rev. B 59, 1758 (1999).

${ }^{25}$ J. P. Perdew, J. A. Chevary, S. H. Vosko, K. A. Jackson, M. R. Pederson, D. J. Singh, and C. Fiolhais, Phys. Rev. B 46, 6671 (1992).

${ }^{26}$ C. Alcock and V. Itkin, Bull. Alloy Phase Diagrams 7, 455 (1986).

${ }^{27}$ K. A. Gschneidner, Bull. Alloy Phase Diagrams 8, 521 (1987).

${ }^{28}$ J. C. Schottmiller, A. J. King, and F. A. Kanda, J. Phys. Chem. 62, 1446 (1958).

${ }^{29}$ B. Predel and F. Sommer, Phys. Condens. Matter 17, 249 (1974).

${ }^{30}$ A. T. Dinsdale, CALPHAD: Comput. Coupling Phase Diagrams Thermochem. 15, 317 (1991).

${ }^{31}$ The criterion for the total energy convergence with respect to different SQS sizes was set to $1 \mathrm{meV} /$ atom. 